\title{
Social Network Analysis of Collaborative Entries for Construction Firms in International Construction Projects
}

\author{
Heedae Park ${ }^{1}$, Woo Yong Jeong ${ }^{2}$ and Seung Heon Han ${ }^{3}$ \\ ${ }^{1}$ Department of Civil Engineering, Yonsei University, Seoul, Korea. PH (822) 2123-2799; FAX \\ 364-5300; e-mail: parkheedae@yonsei.ac.kr \\ ${ }^{2}$ Department of Civil Engineering, Yonsei University, Seoul, Korea. PH (822) 2123-2799; FAX \\ 364-5300; e-mail: trust-jung@gmail.com \\ ${ }^{3}$ Associate Professor, Department of Civil Engineering, Yonsei University, Seoul, Korea. PH \\ 2123-2799; FAX (822) 364-5300; e-mail: shh6018@yonsei.ac.kr
}

\begin{abstract}
The current global construction market is growing tremendously largely due to globalization of world construction markets, rapid development of world-wide communication technologies, extensions of regional Free Trade Blocks, just to name a few. Korean companies, in search for a growth from the recession of domestic construction market, have expanded into the globalized market over the last decades. During this process, collaborative entries have increased remarkably to mitigate the burdens of entry risks. This study focuses on the formation of collaborative networks of Korean contractors when entering foreign markets. Social network analysis (SNA) is introduced to investigate a variety of collaboration patterns and also their impact on performance. To this end, real collaboration cases of 389 overseas projects executed by Korean companies since 1990 were collected and classified into firm's size, collaboration modes, and performance levels. Results of SNA showed a series of apparent tendencies of developing collaboration networks, in order to realize a better performance under the risky project conditions. This study is expected to be used to search a capable partner to gain improved outcome while considering the relevant network hubs as well as their risk levels.
\end{abstract}

\section{Introduction}

Although the current state of world economy causes increasing concerns due to the recent depressed real estate market, financial crisis, and a declined investment on infrastructure, volume of the global construction market has shown a truly remarkable growth over the last decades. In the wake of a global construction market, an increasing number of construction companies have expanded into the global market; not only large companies (LCs) but also small and medium-sized companies (SMCs). Likely, for Korean companies, long-lasting stagnation of domestic construction market leads them to expand into the global market as a way of balanced growth. However, international construction projects involve entry barriers and loads of uncertainties such as political, economic, cultural, legal, and technical risks. These risks can pose a significant threat such that even an internationally-experienced company fails to earn a decent level of profit from risky attempts of overseas projects. In this situation, collaborative entries are remarkably increasing to mitigate the burdens of entry risks that cannot be properly avoided when entering as a single company. Therefore, strategic collaborative formations and joint ventures among companies are on the increase although motives of collaboration modes are different. For example of Korean companies, international contract awarded by collaboration scheme established within LCs is increased from 4 projects in 1996 to 19 projects on 2006. Likewise, those between LC and SMC, and even within SMCs are also continuously increasing from 5 and 0 projects in 1996 up to 73 and 13 projects in 2006, respectively (ICAK, 2007).

With an increase of collaborative entries, previous research on collaboration in construction domain has been widely conducted, mainly focusing on the theoretical principles of partnering and alliancing, and identification of critical factors (Alarcon and Mourgues, 2002; Anvuur and Komaraswamy, 2007; Wong and Cheung, 2005). However, there is little research on collaboration patterns from the network perspective. This study focuses on the formation of collaborative networks between Korean contractors when entering new foreign markets. Social network analysis (SNA) is introduced to investigate a variety of collaboration 
patterns and also their impact on performance. Accordingly, collaboration cases of 389 international construction projects executed by Korean companies from 1990 to 2006 are collected. These cases are then classified into firm's size, collaboration modes, and performance levels using SNA approach. Finally, this study intends to provide a useful direction in establishing business network strategies when forming cooperation with either internationally-experienced or inexperienced companies through collaboration network analysis.

\section{Research Background}

There are a number of researches on firms' collaboration in the construction industry. Anvuur and Kumaraswamy (2007) presented critical influence factors for successful partnering and alliance between construction firms. Wong and Cheung (2005) quantitatively analyzed relationship of factors for partnering through structural equation modeling. In addition, Alarcon and Mourgues (2002) presented success/failure factors of contractor selection in partnering. Lin (2003) also investigated how effectively general contractors can improve relationship with subcontractors. Although these previous researches provided useful knowledge and principles of diverse forms of collaboration, they had limitations in identifying the common aspects and similar patterns from macro-industrial level and network perspective. Therefore, it makes exploring the collaboration networks between firms worth studying to investigate their synergy effects and establish better strategies for fostering collaboration.

Social network analysis (SNA) is a methodology to identify social structures through analyzing interactions and interrelationship of a set of actors (Hu and Rachera, 2008). SNA focuses more on relational properties among components rather than individual attributes. Accordingly, the main objective of SNA is in identifying and analyzing conditions of social relationships' among components (Nooy et al. 2005).

SNA has been applied to investigate various relationships among actors and organizations, and knowledge diffusion in the field of social sciences and economics. Lee (2005), for example, compared development patterns between the automobile industry and semiconductor industry from the network perspective. Hu and Rachera (2008) presented knowledge diffusion network by analyzing co-authorship in the hospitality research domain. Recently, SNA research in the construction field has gradually increased, as construction projects have a wide range of participants that are mutually interrelated and cooperated. Particularly, Pryke (2004) investigated managerial attributes of UK construction projects in connection with procurement modes, supporting the power of SNA application in construction domain. Chinowsky et al. (2008) also proposed an SNA approach to project management that can be used to integrate classic project management concept and knowledge sharing perspective for achieving a higher project performance.

\section{Data Collection and Assumptions}

This study collected international project cases collaboratively performed by Korean companies from 1990 to 2006. Those projects executed by a single entity or contracted for design service were excluded from the analysis; thus, 389 collaborative projects were further analyzed. To categorize the collaboration forms, the authors first used the guideline for distinguishing LCs and SMCs, enacted by the Korean government. According to the criterion, LCs should employ more than 300 full-time workers and hold more than $\$ 2$ million capital. Following table represents the summary of collected data in line with collaboration modes: (1) within LCs, (2) between LC and SMC, and (3) within SMCs. Besides, sample projects are also classified according to the conditions of contracts: joint venture (JV) and prime contractor vs. subcontractor.

Table 1. Summary of Analysis Sample

\begin{tabular}{ccc}
\hline & Joint Venture (JV) & Prime contractor vs. Subcontractor \\
\hline LCs & 10 & 76 \\
LC and SMC & 19 & 231 \\
SMCs & 3 & 50 \\
Total & 32 & 357 \\
\hline
\end{tabular}

This study adopts Pajek® SNA program that enables to visualize and analyze large networks having 
thousands of vertices. Since Pajek ${ }^{\circledR}$ classifies network and color vertices by aid of auxiliary network, the authors discerned profit performances of all projects into dichotomy zones: gain or lose, and similarly, labeled blue and yellow color to LCs and SMCs, respectively. Since this study focuses on the collaboration within Korean companies rather than that with foreign or host country' local companies, this study limited collaboration motives, primarily focusing on the familiarity and mutual-trust between home country's companies, rather than concentrating on the mitigation of entry barrier, reinforcement of bid qualification, and utilization of edged technologies obtainable from foreign firms.

\section{Descriptive Analysis}

Figure 1 shows the collaboration network of 389 entire projects completed by 136 companies. Each circle indicates an actor (contractor), while the line between actors shows an existence of collaboration between two actors. The bigger a circle is, the more a company participates in international projects. The direction of an arrow shows contractual relationships between two actors. If an arrow points B from A, then B implies a subcontractor of A. If there is a parallel contract such as a joint venture, the arrows point to both sides. As shown, actors that have suffered loss are positioned at left side, and vice versa. The brightness of circles classifies LCs (dark actors) and SMCs (bright actors).

In the entire collaboration network, the majority of lines are joined into several actors such as H11, S4, and D7. Interestingly, whereas H11, J8, and D7 in LCs group and their collaborative contractors have realized profits together, D23, S4, and their collaborators have suffered losses. It implies that we can distinguish promising networks from negative ones to achieve a better performance. Thus, it is possible for a particular company to search for probable partners, because good-quality networks, in general, continue to result in respectable performance, and vice versa.

In more details, Figure 2 shows the collaboration network within LCs. Among the actors, five LCs formed the loss-making set but they participated in few projects. There are two types of contractors in the LCs network. Firms such as H11, H15, and S4 collaborated with many companies, whereas other firms including H3, G3 and A20 cooperated with only one company. Interestingly, the collaboration network within LCs pinpoints that more internationally-experienced companies gained higher profits and subsequently they continued to closely collaborate within themselves. For example, H11 and D7, who are the most active companies in this network, gained $5.4 \%$, and $5.5 \%$ of average profit, respectively, posing a higher average profit compared to $1.9 \%$ of other companies. It was found that the two companies continued to collaborate with internationally-experienced firms or allied enterprises belonged to their large conglomerates.

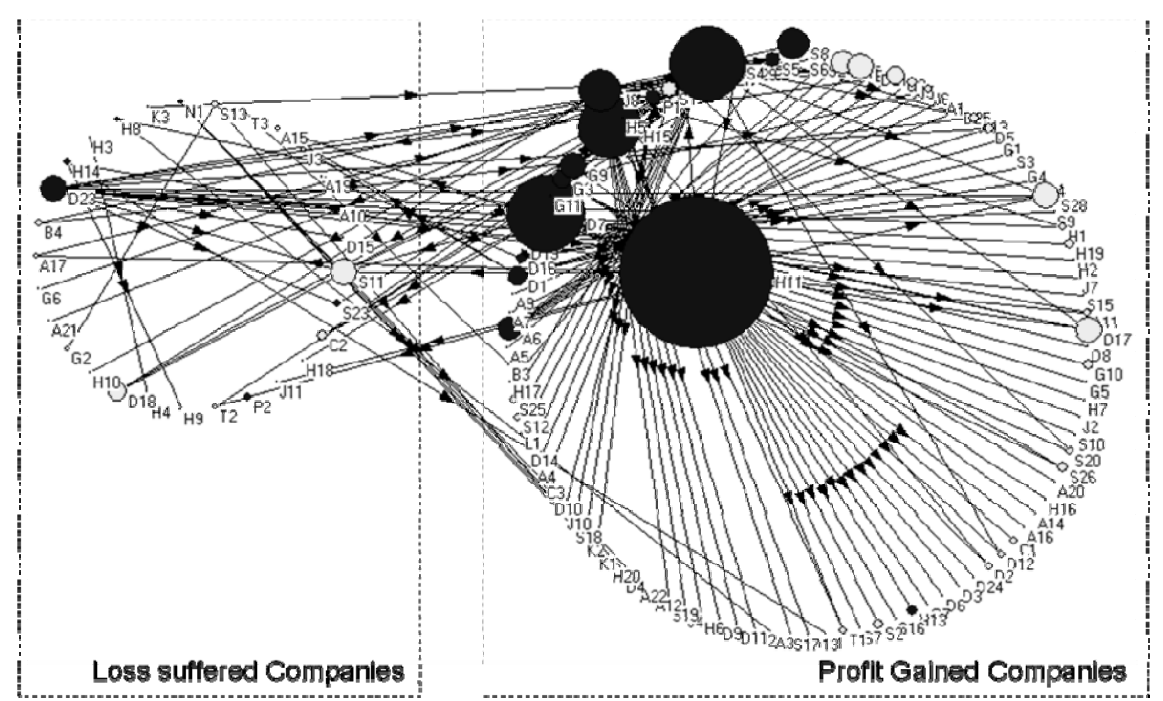

Figure 1. Collaboration Network (All)

The collaboration network between LCs and SMCs shows the most complex relationships where numerous companies work together and collaborate more frequently. In this scheme, the majority is the 
collaboration established between prime contractors (LC) and subcontractors (SMC), occupying 231 out of 250 cases. Only 19 projects were performed through a form of JV where they achieved relatively higher profit than others. It was found that SMCs in this collaboration were chosen as JV partners because they possessed competent skills in construction methods and equipments for building special facilities including power plant, electronic, or subway foundation.

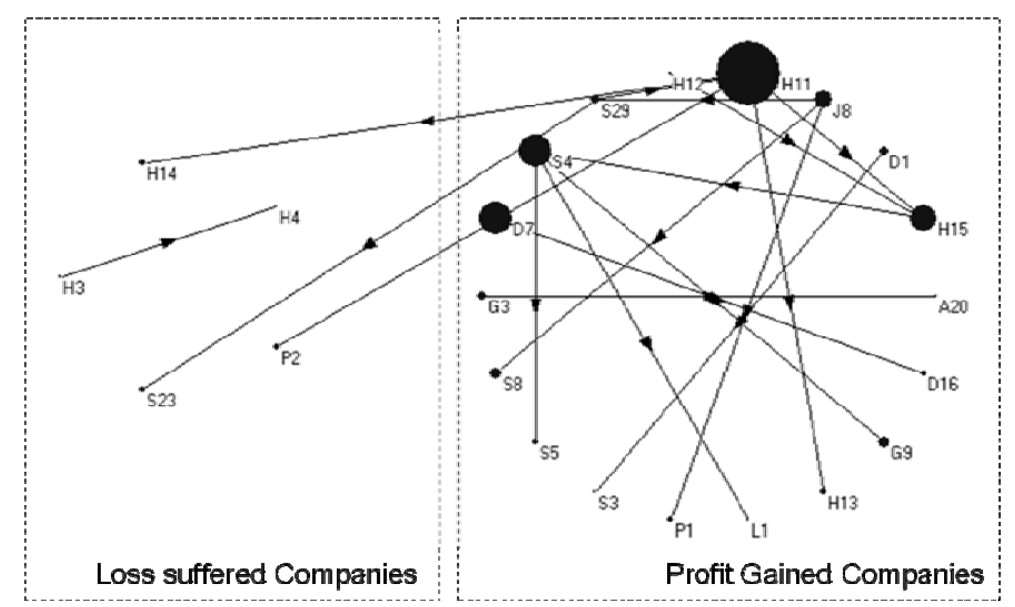

Figure 2. Collaboration Network (between LCs)

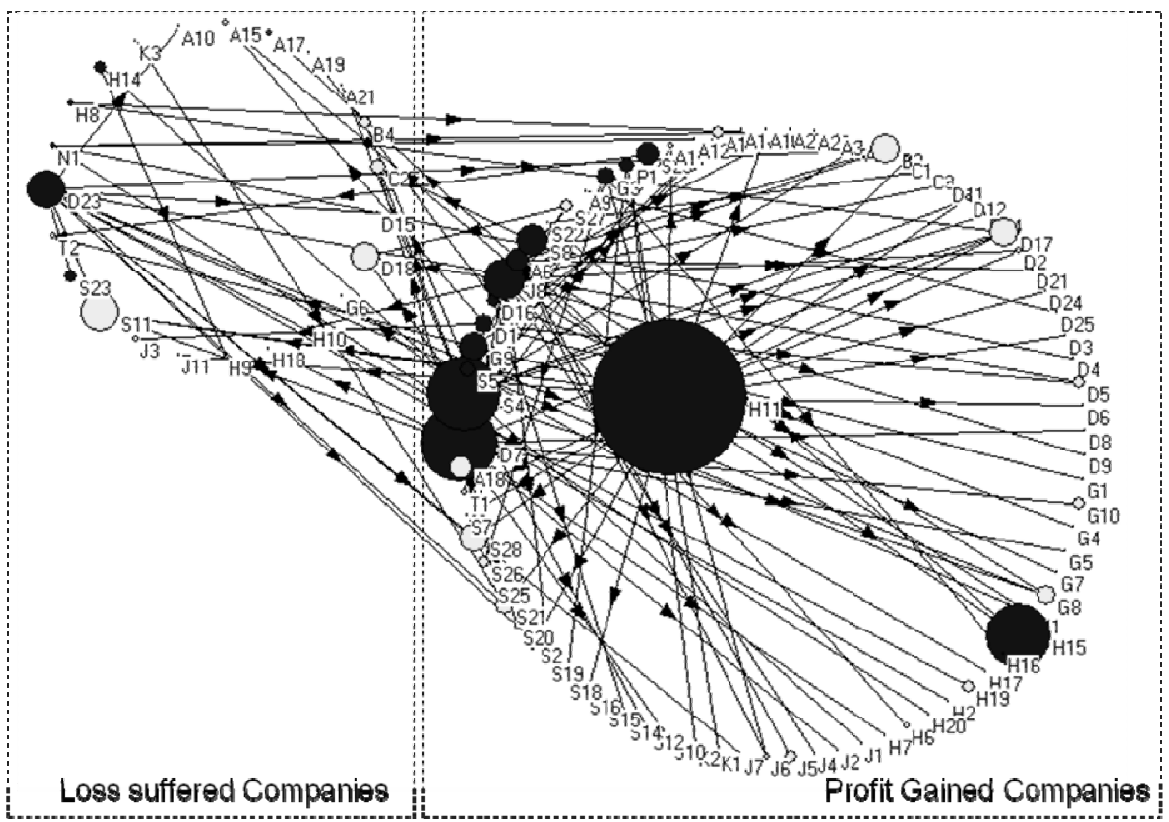

Figure 3. Collaboration Network (between LCs and SMCs)

Meanwhile, SMCs that have collaborated continuously with the same LC gained higher profits than other cases. On the other hand, LCs gained more profit when they have collaborated with many pools of SMCs. More specifically, in the case where LCs collaborated with more than five SMCs, they gained 3.69\% profit on the average, while other cases obtained only $2.62 \%$ profit. This partly explains why LCs prefer to choose SMCs proposing a lower cost among potential bidders rather than engaging a particular subcontractor in their networks. It is fairly paradoxical to the fact that SMCs could gain higher profits through collaborating with a small number of LCs under a quasi-hierarchy system or with a long-term relationship with a particular LC.

Finally, the collaboration network established within SMCs is more segmented than other networks (see Figure 4). Except the few cases of S13, S1, and H5, the most actors were related with only one contractor, implying that they could not maintain capturing project opportunities with this weak network. In another 
aspect, the larger SMCs such as D18, S28, and S21 have obtained many contracts but worked in cooperation with only one company. This is likely because those companies achieved competitive positions with collaborating each other, but it also shows the limitation of SMCs network in developing more capable relationships when entering the international market.

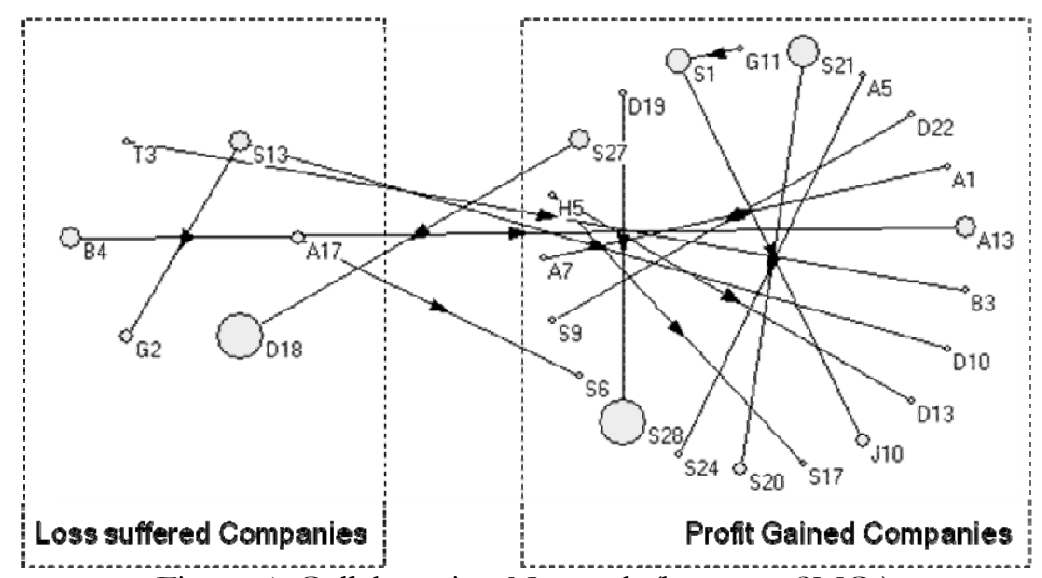

Figure 4. Collaboration Network (between SMCs)

\section{Network Attributes}

SNA displays not only a visual representation but also network variables that express structural attributes, which in turn helps analyzing a network in further details. This paper investigates degree, density, and centrality through examining network variables. Also, several other properties are investigated for comprehensive understanding of networks.

\section{Degree, Density, and Centrality}

Degree indicates the number of actors collaborating with a specific actor, and it is a good index to measure regional centrality. Density represents a level of crowd; thus, is calculated by dividing the number of existing lines with the number of all possible lines, therefore, density varies from 0 (no connection in the network) to 1 (every vertex are interrelated). Lastly, centrality denotes the grade of structural efficiency. There are three types of centrality whether the focus is placed on inward directive, outward directive, and total connection : indegree centrality, outdegree centrality, and total centrality. Equations of three attributes above are like following.

$$
\begin{aligned}
& \text { Degree }=\text { number of connected vertices to a vertex } \\
& \text { Density }=l /\left(n^{*}(n-1)\right)(l=\text { number of existent lines, } n=\text { number of existent vertices) } \\
& \text { Centrality }=(\text { variation in the degree of vertices }) / \text { (maximum degree variation) }
\end{aligned}
$$

Table 2 shows the summary of each networks' attributes. Despite the low level of density as a whole, the networks established within both LCs and SMCs are more intense, because density is inversely proportional to a network size. Meanwhile, networks within LCs, and between LC and SMC have higher centrality, particularly showing a tendency of outdegree centrality. This implies that there exist a small number of large contractors connected with many subcontractors such that networks tend to migrate toward several hubs. By contrast, the centrality of SMCs network shows the lowest value in all, as the SMCs' network is fairly segmented and scattered.

\section{Frequency Distribution and Structural Equivalence}

This paper analyzes two more attributes: frequency distribution and structural equivalence. The former refers to a summary of social oftenness to extend a cooperation cluster, and the latter represents the hierarchical formation of relationship among all actors in a network. As shown in Table 3, most contractors, around $52 \% \sim 78 \%$, are found to be collaborated with only one contractor in every network. The most sociable contractors within LCs, and between LC and SMC built up huge clusters engaged with 18 and 28 contractors, respectively, whereas that of SMCs network constitutes a small cluster with only 3 contractors. However, as previously mentioned, there is a weak evidence to connect between how sociable a contractor is 
and whether a contractor could make profits. It rather relies on how experienced a companion contractor is and whether a contractor establishes a collaborative network with a firm that has demonstrated a better performance.

Table 2. Summary of Network Attributes

\begin{tabular}{ccccccccc}
\hline & Degree & \multirow{2}{*}{ Density } & \multicolumn{3}{c}{ Centrality } & \multicolumn{3}{c}{$\%$ of firms gaining profit } \\
& $(\mathrm{min}-\mathrm{max})$ & & In & Out & All & LCs & SMC & All \\
\hline LCs & $1-18$ & 0.06 & 0.05 & 0.15 & 0.08 & 78.3 & - & 78.3 \\
LC and SMC & $1-28$ & 0.01 & 0.02 & 0.20 & 0.09 & 69.6 & 80.2 & 77.9 \\
SMCs & $1-3$ & 0.02 & 0.01 & 0.05 & 0.01 & - & 77.8 & 77.8 \\
\hline
\end{tabular}

Table 3. Frequency Distribution of Cluster Numbers

\begin{tabular}{|c|c|c|c|c|c|c|}
\hline \multirow{2}{*}{ Cluster } & \multicolumn{2}{|c|}{ LCs } & \multicolumn{2}{|c|}{$\mathrm{LC}$ and $\mathrm{SMC}$} & \multicolumn{2}{|c|}{ SMCs } \\
\hline & Freq. & Cum.Freq. $\%$ & Freq. & Cum.Freq. $\%$ & Freq. & Cum.Freq. $\%$ \\
\hline 1 & 15 & 65.2 & 55 & 52.9 & 21 & 77.8 \\
\hline 2 & - & 65.2 & 19 & 71.2 & 3 & 88.9 \\
\hline 3 & 5 & 86.9 & 11 & 81.7 & 3 & 100.0 \\
\hline 4 & 1 & 91.3 & 4 & 85.6 & - & \\
\hline 5 & - & 91.3 & 2 & 87.5 & - & \\
\hline & & & & & - & \\
\hline 14 & 1 & 95.7 & - & 94.2 & - & \\
\hline & & & & & - & \\
\hline 18 & 1 & 100.0 & - & 96.2 & - & \\
\hline & - & & & & - & \\
\hline 21 & - & & 2 & 99.0 & - & \\
\hline 28 & - & & 1 & 100.0 & - & \\
\hline Sum & 23 & - & 104 & - & 27 & - \\
\hline
\end{tabular}

Figure 5(a) and Figure 5(b) represent the structural equivalence of LCs network and SMCs network, respectively. Diagram of network between LCs and SMCs is not included here for the sake of brevity. Since the SMCs' network is broken down into lots of pairs, it has a simple and uniform structure, while those within LCs, and between LC and SMCs have perpendicular and hierarchical structure. Based on the analysis results, the authors conclude that searching an experienced companion in the LCs' network is relatively easy, but it is not the case in the SMCs' network because capable contractors are not clearly disclosed in this type of system's structure.

\section{Conclusion}

This study focuses on the formation of collaborative networks established within Korean companies when expanding into new foreign markets. The authors analyzed 398 cases of international projects executed by such collaborative organizations as JV and subcontracting. SNA was introduced to investigate a variety of collaboration patterns and also their impact on the level of performance. In this process, the authors attempted to relate the networks with their profit performances, in association with collaboration modes. Results of SNA emphasized that; (1) within the LCs' network, making continuous collaboration with a particular company intended to result in higher profits, (2) between LCs and SMCs network, SMC could realize a higher profit when making a continuous collaboration with the same LC, whereas LCs gained a less profit when working with one particular SMC, (3) if SMCs failed to achieve a competitive edge in international construction per sea, they were difficult to find a promising network and so to gain a better profit, and (4) the more hierarchical the structural equivalence, the higher the possibility for searching excellent partners to gain improved profit. Recalling that the main scope of this research is to analyze 
collaborations made within home country's companies, future procedural research will focus on more broadened areas including diversities of collaboration patterns, regional distributions, and project types, in order to explore and understand how these factors influence network conditions and performance. Through an extension of current analysis, construction firms can effectively choose a suitable entry partner to capture more overseas project opportunities and better results while considering the relevant network hubs as well as their risk levels.

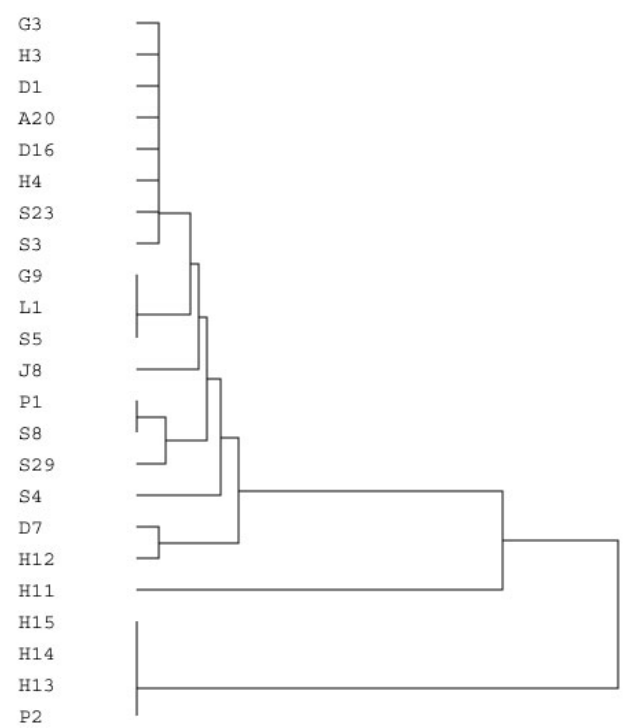

(a)

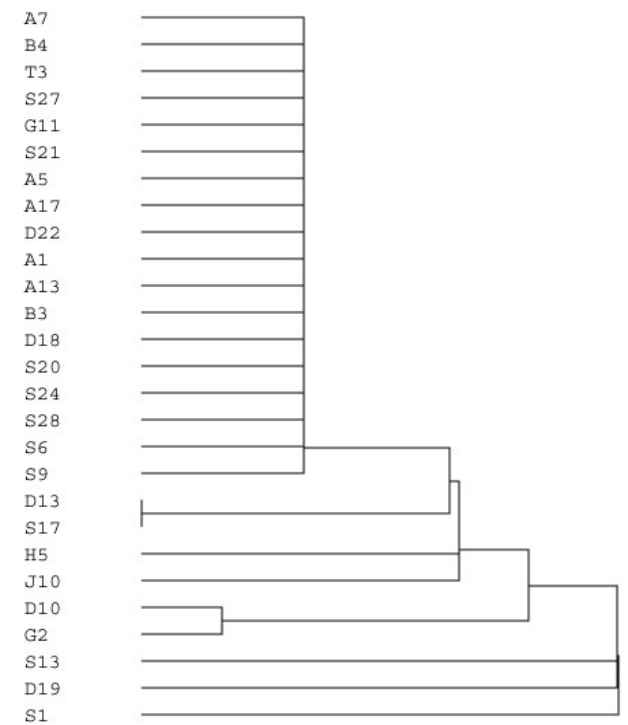

(b)

Figure 5. Structural Equivalence ((a): LCs, (b) SMCs)

\section{Acknowledgement}

This research was supported by a grant (07 Urban Renaissance B03) from High-Tech Urban Development Program funded by the Ministry of Construction and Transportation of Korea.

\section{References}

[1] Alarcon, L. F. and Mourgues, C. (2002). "Performance Modeling for Contractor Selection." J. Manage. Eng., 18(2), 52-60.

[2] Anvuur, A. M. and Kumaraswamy, M. M. (2007). "Conceptual Model of Partnering and Alliancing." J. Const. Eng. and Manage., 133(3), 225-234.

[3] Chinowsky, P., Diekmann, J., and Galotti, V. (2008). "Social Network Model of Construction." J. Const. Eng.and Manage., 134(10), 804-812.

[4] Hu, C. and Rachera, P. (2008). "Visual Representation of Knowledge Networks: A Social Network Analysis of Hospitality Research Domain.” Int'l J. Hospitality Manage., 27, 302-312.

[5] International Contractors Association of Korea (ICAK) (2008) "International Construction Information Service: Summary of the present state.” <http://www.icak.or.kr/sta/sta_0101.php> (Dec. $8,2008)$.

[6] Lee, G. H. (2005). "Mutual Influence of Corporate Networks and Industrial Change.” J. of Korea Society of Social Science, 39(4), 1-40.

[7] Lin, P. H. (2003). "Decision Support for Subcontracting Procurement Based on Multi-Attribute Utility Theories." Const. Research Congress 2003, Honolulu, Hawaii, ASCE.

[8] Ministry of Land, Transportation, and Maritime Affairs (2007). "Development of Pilot Program for Collaboration of Large Enterprise and Small-Medium Enterprise." Research Report, No. 2007-03.

[9] Nooy, W., Mrvar, A., and Batageli, V. (2005). "Exploratory Social Network Analysis with Pajek." Cambridge University Press, Cambridge, UK.

[10] Pryke, S. D. (2004). "Analysing construction project coalitions: exploring the application of social network analysis." Constr. Manage. and Economics, 22(8), 787-797. 